\title{
Research Article \\ IMPORTANCE OF PLATELET COUNT IN DIAGNOSIS OF DENGUE INFECTION
}

\author{
NAIK T.B., MURUGESH K. ${ }^{*}$ AND EUPHEMIA ZENITH
}

Department of Microbiology, Chamarajanagar Institute of Medical Sciences, Chamarajanagar, 571313, Rajiv Gandhi University of Health Sciences, Bengaluru, 560041

*Corresponding Author: Email- murugesh2974@gmail.com

Received: June 11, 2018; Revised: June 18, 2018; Accepted: June 19, 2018; Published: June 30, 2018

\begin{abstract}
Introduction: Dengue is an acute, potentially fatal viral infection that can culminate into dengue hemorrhagic fever and dengue shock syndrome. It has emerged as a notable public health problem in recent decades in terms of the mortality and morbidity associated with it. Specific antibody $\lg M$ and $\lg G$ detection along with the newer parameter NS1 antigen detection has been the main stay of diagnosis. Apart from these parameters, thrombocytopenia is a constant finding and therefore platelet count can be used as an accessory test for diagnosis of dengue infection in the laboratories where resources are limited. Materials and Methods: A cross-sectional study was conducted using secondary data of clinically suspected dengue cases reported to the microbiology laboratory during June-August months of 2017 at CIMS teaching hospital, Chamarajanagar. Samples were tested either for NS1or IgM by ELISA depending on duration of fever and platelet counts were obtained. Statistical analysis was done using WHO Epi info software version 3.5.4. Results: Out of 1653 clinically suspected cases, 265(16.03\%) were found to be positive for dengue infection either by NS1 or IgM ELISA. Among them $135(50.95 \%)$ were females and majority 211 (79.62) were below the age of 30 years. Proportion of thrombocytopenia was significantly higher in dengue positive cases than in negatives. Conclusion: In resource limited setting thrombocytopenia can be used as predictor of dengue infection for early initiation of therapy to reduce the morbidity and mortality associated with it.
\end{abstract}

Keywords- Dengue, IgM, NS1, Platelet count

Citation: Naik T.B., et al., (2018) Importance of Platelet Count in Diagnosis of Dengue Infection. International Journal of Microbiology Research, ISSN: 0975-5276 \& E-ISSN: 09759174, Volume 10, Issue 6, pp.-1264-1267.

Copyright: Copyright@2018 Naik T.B., et al., This is an open-access article distributed under the terms of the Creative Commons Attribution License, which permits unrestricted use, distribution and reproduction in any medium, provided the original author and source are credited.

\section{Introduction}

Dengue is an acute, potentially fatal viral infection that can culminate into dengue hemorrhagic fever (DHF) and dengue shock syndrome (DSS). It is caused by five serotypes of dengue virus (DV), namely DEN-1, DEN-2, DEN-3, DEN-4 and DEN5 [1] belonging to genus Flavivirus and family Flaviviridae. It spreads through the bite of infected Aedes aegypti mosquito. Most of the primary infections are uneventful. Infection with one serotype confers an individual life-long immunity to that serotype and cross-reactivity to the other serotypes, which is the basis for the complications like DHF and DSS [2]. Dengue affects up to 100 million people annually, with 5,00,000 cases of DHF/DSS leading to around 30,000 deaths, mostly among children [3]. It is almost endemic throughout India. Due to rapid urbanization, lifestyle changes and poor water management techniques, the risk of dengue infection in India has increased in recent years [4]. Dengvaxia( $R$ ) is a live attenuated tetravalent vaccine for dengue that is currently under evaluation in phase 3 clinical trials in Asia (Indonesia, Malaysia, Philippines, Thailand and Vietnam) and Latin America (Brazil, Colombia, Honduras, Mexico and Puerto Rico). The protective efficacy of Dengvaxia( $R$ ) has been estimated to range between $50.2 \%$ and $76.6 \%$ for different ages and serotypes [5]. It is well known that early and specific diagnosis of DHF or DSS followed by supportive therapy reduces morbidity and mortality [6]. Virus isolation in cell culture, identification of viral genomic sequence by nucleic acid amplification techniques like RT-PCR, NASBA (Nucleic acid based amplification) and detection of dengue specific lgM, IgG antibodies by ELISA (Enzyme linked immunosorbent assay) or by immunochromatographic methods are being used currently in the laboratories for diagnosis of dengue infection [7]. Virus isolation and molecular techniques cannot be used as routine diagnostic tests because they are laborious, time consuming and require specialized laboratory facilities [8]. Serological diagnosis can be done only after appearance of antibodies which usually takes 4-6 days of illness [9]. Combination of NS1 (Non structural protein) antigen detection along with antibody detection increases the diagnostic rates as secretory protein NS1 antigen is seen in high concentrations during acute phase of illness ( 1 to 5 days) [10,11]. Immunochromatographic detection of these serological markers yield rapid results but have low sensitivity as compared to ELISA [12]. During febrile period thrombocytopenia starts and during DHF platelet count reduces progressively.[13]. A drop in platelet count below $1,00,000$ per $\mathrm{mm}^{3}$ is usually found between the third and eighth day of illness as per WHO guidelines, so thrombocytopenia can be used to detect and monitor DHF.[14] Platelet count can be roughly estimated by microscopy even in the peripheral laboratories and it is the only accessory laboratory test which supports the diagnosis of dengue [15] Further the resource poor health care system has to depend upon simple to perform and easy to interpret laboratory tests for diagnosis. With this background we tried to correlate the dengue serological markers with platelet counts.

\section{Objectives}

To find the seroprevalence of dengue infection in the study setting.

To find out the association between platelet count and serological markers (NS1ag and $\lg \mathrm{M}$ ) in the acute stage of dengue infection.

\section{Materials and Methods}

A cross-sectional study was conducted using secondary data of clinically suspected dengue cases who attended various clinical departments of our hospital and reported to the microbiology laboratory during June-August months of 2017 at tertiary care hospital of Chamarajanagar Institute of Medical Sciences, Chamarajanagar. Permission for the study was taken from the head of the Institute. As per WHO guidelines samples of patient $<5$ days of fever were tested for NS1Ag by Tulipqualisa and $\geq 5$ days of fever for lgM by MAC ELISA, developed by NIV (National Institute of Virology), Pune. 
Manufacturer's instructions were followed strictly while performing the ELISA. Platelet counts of the suspected patients were recorded, irrespective of positive or negative result for the above test.

\section{Seroassays}

Approximately $5 \mathrm{ml}$ of blood samples were collected from all the suspects of Dengue as a part of the routine laboratory work and the sera were separated and tested for the Dengue NS1 and lgM by ELISA methods.

\section{NS1 Ag assay}

NS1Ag tulipqualisa test kit was used to perform the test. NS1Ag tulipqualisa is a solid phase enzyme linked immunosorbent assay (ELISA) based on the "Direct Sandwich" principle. Anti-Dengue NS1 antibodies were coated on microwells with high reactivity for Dengue NS1 antigen. After adding the samples to the wells, enzyme conjugate (monoclonal anti-dengue NS1 antibodies linked to Horseradish peroxidase (HRPO) were added. A sandwich complex was formed in the well wherein dengue NS1 (from serum sample) is "trapped" or "sandwiched" between the antibody and antibody HRPO conjugate. Wash buffer was added that will wash off unbound conjugate. The amount of bound peroxidase was proportional to the concentration of dengue NS1 antigen present in the sample. Blue colour developed on addition of the substrate buffer and chromogen. The intensity of colour was proportional to the concentration of dengue NS1 antigen. To limit the enzyme-substrate reaction stop solution was added, and yellow colour which developed was read at $450 \mathrm{~nm}$ spectrophotometrically. Results were interpreted as per the manufacturer's guidelines.

\section{Detection of $\operatorname{lgM}$}

Samples of patient $\geq 5$ days of fever were tested for the presence of dengue specific IgM antibodies by using MAC ELISA, developed and commercialized by NIV (National Institute of Virology), Pune, and recommended by National Vector Borne Disease control programme. Tests were done and results were read as per the literature provided.

\section{Platelet Count}

EDTA blood samples were collected and the platelet count was done by automated analyzer and cross checked by light microscope and interpreted as normal, when the count was between $1,50,000-4,50,000 / \mathrm{mm}^{3}$ and DHF, when the count was $<1,00,000 / \mathrm{mm}^{3}$ (WHO cut off for platelet count for DHF). Statistical analysis: Statistical analysis was done using WHO Epi info software version 3.5.4.

\section{Results}

[Table-1] Diagnosis of dengue infection by dengue specific parameters. Out of total 1653 samples tested, 751 were tested for NS1only and 902 samples were tested for IgM only depending on the stage of illness. Out of 1653 clinically suspected cases, $265(16.03 \%)$ were found to be positive either by NS1 or by IgM ELISA. Rate of seropositivity was $228(30.35 \%)$ for NS1 and for IgM was 37 $(4.10 \%)$ as evident from the table. [Table-2] and [Fig-1] shows gender wise distribution of Dengue suspects and positives. Out of the total 265 dengue positive cases, $130(49.05 \%)$ were males and $135(50.95 \%)$ were females. Of the 228 positive cases detected by NS1, $111(48.68 \%)$ were males and $117(51.31 \%)$ were females. Similarly, out of the total 37 positive cases diagnosed by lgM, 19 $(51.35 \%)$ were males and the rest 18 (48.64\%) were females. [Table-3] and [Fig-2] shows age wise distribution of Dengue suspects and positives. Of the 265 total dengue positive cases, majority i.e., 211 (79.62) were below the age of 30 years. Similarly, majority i.e., 184 (80.7\%) of cases detected by NS1 and majority i.e., $27(72.97 \%)$ of cases detected by lgM were in the age group $<30$ years. [Table-4] Association of dengue cases (detected by NS1) with thrombocytopenia was assessed as per the objective of the study. Thrombocytopenia was found in 162 $(71.05 \%)$ of positive cases against only $152(29.06 \%)$ of negatives and this difference was found to be statistically significant ( $p$ value $<0.0001$ ). [Table-4] shows association of dengue cases (detected by $\operatorname{lgM}$ ) with thrombocytopenia. When IgM antibodies were considered for the diagnosis of dengue infection, thrombocytopenia was seen in 24 of $37(64.86 \%)$ positive cases and in only 216
$(24.97 \%)$ of negatives and it was found to be statistically significant ( $p$ value= $0.01)$.

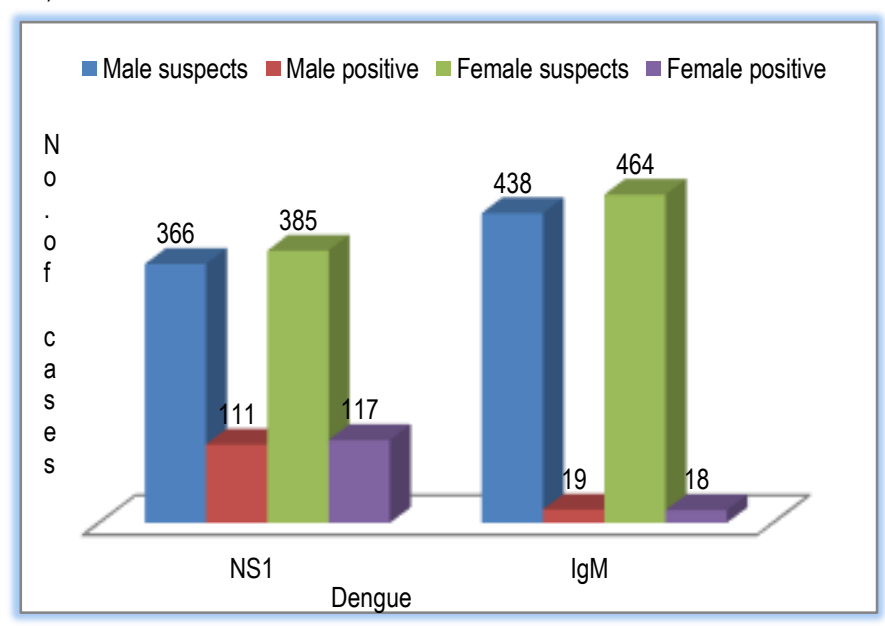

Fig-1 Gender wise distribution of dengue suspects and positives

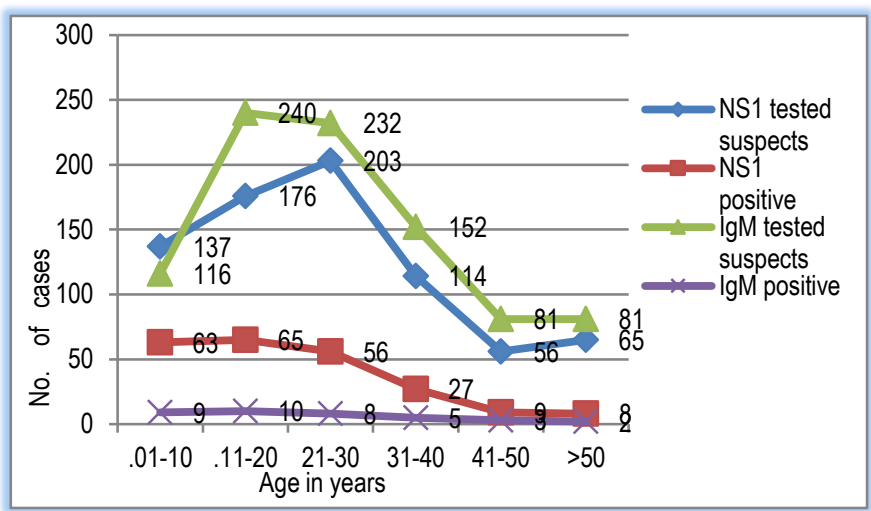

Fig-2 Age wise distribution of dengue suspects and positives

Table-1 Diagnosis of Dengue by dengue specific parameters

\begin{tabular}{|c|c|c|c|}
\hline Parameters & Suspects tested & Positive & Rate of positivity \\
\hline & No. & No. & $(\%)$ \\
\hline NS1 only & 751 & 228 & 30.35 \\
\hline IgM only & 902 & 37 & 4.1 \\
\hline Total & 1653 & 265 & 16.03 \\
\hline
\end{tabular}

Table-4 Association of Dengue status (using NS1ag test) with thrombocytopenia

\begin{tabular}{|c|c|c|c|}
\hline Thrombocytopenia & \multicolumn{3}{|c|}{ Dengue NS1 antigen } \\
\hline & Positive & Negative & Total \\
\hline & No. & No. $\%$ & No. \\
\hline Present & $162(71.05 \%)$ & $152(29.06 \%)$ & 314 \\
\hline Absent & $66 \quad(28.94 \%)$ & $371 \quad(70.93 \%)$ & 437 \\
\hline Total & $228(100 \%)$ & $523(100 \%)$ & 751 \\
\hline \multicolumn{4}{|c|}{$x^{2}=115.06, p$ value $<0.0001$} \\
\hline
\end{tabular}

Table-5 Association of Dengue status (using $\lg M$ test) with thrombocytopenia

\begin{tabular}{|c|cc|cc|c|}
\hline Thrombocytopenia & \multicolumn{4}{|c|}{ Positive } & \multicolumn{2}{c|}{ Negative } & Total \\
\hline & 24 & $(64.86 \%)$ & 216 & $(24.97 \%)$ & 240 \\
\hline Present & 13 & $(35.13 \%)$ & 649 & $(75.29 \%)$ & 662 \\
\hline Absent & 37 & $(100 \%)$ & 865 & $(100 \%)$ & 902 \\
\hline Total & \multicolumn{5}{|c}{$\varkappa^{2}=6.359, p$ value $=0.01$} \\
\hline
\end{tabular}

\section{Discussion}

Dengue is the most important arthropod-borne viral disease of public health significance for the past few decades. Early diagnosis of dengue is of utmost importance to reduce the morbidity and mortality associated with it, especially in the peripheries. Apart from dengue specific parameters, platelet count can be used in remote areas to support the diagnosis. 
Naik T.B., Murugesh K. and Euphemia Zenith

Table-2 Gender wise distribution of Dengue suspects and positives

\begin{tabular}{|c|c|c|c|c|c|c|}
\hline \multirow{2}{*}{ Gender } & $\begin{array}{c}\text { Total } \\
\text { Suspects }\end{array}$ & $\begin{array}{c}\text { Total } \\
\text { Positive }\end{array}$ & $\begin{array}{c}\text { Suspects } \\
\text { tested for NS1 }\end{array}$ & $\begin{array}{c}\text { NS1 } \\
\text { (positive) }\end{array}$ & $\begin{array}{c}\text { Suspects } \\
\text { tested for lgM }\end{array}$ & $\begin{array}{c}\text { IgM } \\
\text { (positive) }\end{array}$ \\
\hline & No. & No (\%) & No. & No (\%) & No. & No (\%) \\
\hline Male & 792 & $130(49.05 \%)$ & 366 & $111(48.68 \%)$ & 438 & $19(51.35 \%)$ \\
\hline Female & 861 & $135(50.94 \%)$ & 385 & $117(51.31 \%)$ & 464 & $18(48.64 \%)$ \\
\hline Total & 1653 & $265(100 \%)$ & 751 & $228(100 \%)$ & 902 & $37(100 \%)$ \\
\hline
\end{tabular}

Table-3 Age wise distribution of Dengue suspects and positives

\begin{tabular}{|c|c|c|c|c|c|c|}
\hline Age (Yrs) & $\begin{array}{c}\text { Total } \\
\text { Suspects }\end{array}$ & Total Positive & $\begin{array}{l}\text { Suspects tested } \\
\text { for NS1 }\end{array}$ & $\begin{array}{c}\text { NS1 } \\
\text { (positive) }\end{array}$ & $\begin{array}{l}\text { Suspects tested } \\
\text { for lgM }\end{array}$ & $\begin{array}{c}\lg \mathrm{M} \\
\text { (positive) }\end{array}$ \\
\hline & No. & No $\%$ & No. & No $\%$ & No. & No $\%$ \\
\hline $1-10$ & 253 & $72(27.16)$ & 137 & $63(27.63)$ & 116 & $9(24.32)$ \\
\hline $11-20$ & 416 & 75 (28.3) & 176 & $65(28.5)$ & 240 & $10(27.02)$ \\
\hline $21-30$ & 435 & $64(24.15)$ & 203 & $56(24.56)$ & 232 & $8(21.62)$ \\
\hline $31-40$ & 266 & $32(12.07)$ & 114 & $27(11.84)$ & 152 & $5(13.51)$ \\
\hline $41-50$ & 137 & $12(4.52)$ & 56 & $9(3.94)$ & 81 & $3(8.1)$ \\
\hline $51-60$ & 146 & $10(3.77)$ & 65 & $8(3.5)$ & 81 & $2(5.4)$ \\
\hline Total & 1653 & $265(100)$ & 751 & $228(100)$ & 902 & $37(100)$ \\
\hline
\end{tabular}

The present study was undertaken to study the seroprevalence of dengue infection and to test association of dengue seromarkers with platelet count. In the present study out of 1653 clinically suspected dengue cases, 265 (16.03\%) were found to be positive for dengue infection either by NS1 or IgM ELISA. Various studies have reported a seroprevalence level ranging from $10 \%-21 \%[2,15-18]$. On the contrary, few other studies have found a lesser seroprevalence values $[18,19]$ and some others have reported higher values than our study $[8,21-24]$. Of the 751 cases tested for NS1 antigen only, the rate of seropositivity was 228 (30.35\%). This finding is consistent with the studies done by Kulkarni, et al., [2] in which 30\%, Agarwal, et al., 29.41\% [16], Neralwar, et al., 29\% [20], Mehta, et al., $29.63 \%$ [21] and Datta, et al., 23.3\% [25] of cases were positive for NS1 antigen only. In contrast some studies have reported higher seropositivity for NS1 antigen $[8,22,23]$ while other have reported lesser seropositivity $[18,24,26]$. On the other hand out of 902 tested for IgM only 37 (4.10\%) cases were seropositive. Similar seropositivity rate was found in the study conducted by Gitanjali, et al. in which 4.7\% [22], Sindhanai, et al.,10\% [23], Lakshmi, et al., 5\% [24], patients were serologically positive for dengue infection. Higher values were found in other studies $[2,8,16,18,20,21]$. Of the 265 total positive cases of dengue, the highest numbers of cases were found in the age group of 1-30 years similar to the finding from other studies $[2,20,27,28]$. Comparatively lower immunity and intrinsically more permeable vascular endothelium in children render them more susceptible to dengue infection. Similarly, out of the total positive cases, $130(49.05 \%)$ were males and the rest $135(50.94 \%)$ were females contrary to the findings from other studies $[18,20,21]$. Thrombocytopenia in dengue infection is not an early indicator of severe disease but it helps in predicting the progression of disease and the present study tried to find the association of dengue positivity with thrombocytopenia. Thrombocytopenia was evident in $162(71.05 \%)$ of positive cases as against only $152(29.06 \%)$ of negative cases detected by NS1and it was in consistent with other studies [2,21-23]. Similarly, thrombocytopenia was more prevalent in positives i.e., 24 of $37(64.86 \%)$ than in negatives $216(24.97 \%)$ when diagnosed by $\operatorname{lgM}$ in the present study similar to the findings of other studies $[2,21,23]$ The above results were statistically significant.

\section{Conclusion}

The present study highlights dengue infection rate in this part of state and its predominance in economically productive age groups. We found that thrombocytopenia was evident in dengue positive cases than in dengue negative cases.

Application of Research: Thrombocytopenia can be used as predictor of dengue infection for early initiation of therapy to reduce the morbidity and mortality associated with it, especially in poor resource settings. Such predictions also help to initiate preventive/ control measures well in time for the containment of spread of the disease.
Research Category: Diagnosis of Dengue

\author{
Abbreviations \\ IgM- Immunoglobulin M \\ Ig G-Immunoglobulin G \\ NS1- Non structural protein 1 \\ CIMS- Chamarajanagar Institute of Medical Sciences \\ ELISA-Enzyme linked immunosorbent assay \\ DHF- Dengue hemmoraghic fever \\ DSS- Dengue shock syndrome \\ DV- Dengue virus \\ RT- PCR- Reverse transcriptase polymerase chain reaction \\ NASBA- Nucleic acid based amplification \\ WHO- World health organization \\ HRPO- Horse radish peroxidase \\ NIV- National institute of Virology \\ EDTA- Ethylene diamine tetra acetic acid
}

Acknowledgement / Funding: Author thankful to Chamarajanagar Institute of Medical Sciences, Chamarajanagar, 571313, Rajiv Gandhi University of Health Sciences, Bengaluru, 560041, Karnataka India

\section{${ }^{*}$ Research Guide or Chairperson of research: Dr Murugesh K}

University: Rajiv Gandhi University of Health Sciences, Bengaluru, 560041

Research project name or number: Nil

\section{Author Contributions: All author equally contributed}

Author statement: All authors read, reviewed, agree and approved the final manuscript

\section{Conflict of Interest: None declared}

Ethical approval: This article does not contain any studies with human participants or animals performed by any of the authors.

\section{References}

[1] Mustafa M.S., Rastogi V., Jain S., Gupta V. (2015) Medical Journal Armed Forces of India, 7, 67-70.

[2] Kulkarni R.D., Patil S.S., Ajantha G.S., Upadhya A.K., Kalabhavi A.S., Subhada R.M. et al., (2011) Indian Journal of Medical Microbiology, 29(4),359-62.

[3] World Health Organization. Dengue haemorrhagic fever: Diagnosis, treatment, prevention and control (1997) $2^{\text {nd }}$ edition. Geneva, Switzerland: Chapter 2, Clinical Diagnosis, 12-23. 
[4] Mairuhu A.T., Wagenaar.J., Brandjes D.P., Van Gorp E.C. (2004) Eur. J. Clin. Microbiol. Infect. Dis. 23, 425-33.

[5] Mutheneni S.R., Morse A. P., Caminade C., Upadhyayula S. M. (2017) Emerging Microbes and Infections, 6, e70.

[6] Peters C.J. (2008) Infections caused by arthropod and rodent borne viruses. In: Fauci AS, editor. Harrison's principles of internal medicine. 17th ed. New York: Mcgraw Hill Medical Publishing Division, 122639.

[7] Vijayakumar T.S., Chandy S., Sathish N., Abraham M., Abraham P., Sridharan G. (2005) Indian J Med Res, 121,100-107.

[8] Santosh S.T., Chincholkar V.V., Kulkarni D.M., Nilekar S.L., Ovhal R.S., Halgarkar C.S. (2013) Int. J. Curr. Microbiol. App. Sci., 2(12), 4044.

[9] Young P.R., Hilditch P.A., Bletchly C., Halloran W. (2000) J Clin Microbiol, 38,1053-7.

[10] Chakravarti A., Kumar A., Malik S. (2011) Southeast Asian J Trop Med Public Health, 42(2),297- 302.

[11] Fry S.R., Meyer M., Semple M.G., Simmons C.P., Sekaran S.D., Huang J.X., et al., (2011) PLOS neglected tropical diseases, 5(6), 1199.

[12] Halstead S.B., Udomsakdi S., Singharaj P., Nisalak A. (1969) Am J Trop Med Hyg, 18, 984-96.

[13] Pimpan P., Prasert T. (1993) WHO SEARO, 62-71.

[14] World Health Organization (1997) Dengue haemorrhagic fever: Diagnosis, treatment, prevention and control. $2^{\text {nd }}$ edition. Geneva, Switzerland. Chapter 4, Laboratory Diagnosis, 35-47.

[15] Jyothi P., Metri BC (2015) Adv Biomed Res, 4, 26.

[16] Agarwal S.G., Rukadikar A.R., Laghawe A., Tripathi A. (2014) J of Evolution of Med and Dent Sci, 3(10), 2543-2547

[17] Pruthvi D., Shashikala P., Shenoy V. (2012) J Blood Disorders Transf, 3(4), 1-4.

[18] Biradar A.M., Nadagir D.S., Shankar M.K., Naik T.B. (2016) Int. J. Curr. Microbiol. App. Sci, 5(9), 725-732

[19] Wadekar M. D., Naik T. B., Upadhya A. K., Swarooparani N.B. (2016) International Journal of Microbiology Research, 8(1), 720-722.

[20] Neralwar A., Banjare B., Barapatre R. (2015) Int J Res Med Sci 3, 2826-30.

[21] Mehta K.D., Ghediya B., Sheth S., Khandhediya S., Shingala H., Sinha M. (2016) National Journal of Laboratory Medicine, 5(2), 55-59.

[22] Badave G.K., SaiSwaroop P., Rao P.N. (2015) J. Curr. Microbiol. App. Sci, 3, 779-784

[23] Sindhanai V., Banoo S., Rajkumar N., Chander V.C.S. (2016) Sch. J. App. Med. Sci, 4(2), 618-622

[24] Lakshmi P.S., Nainar P. (2014) Journal of the Scientific Society, 41(2), 85-88.

[25] Datta S., Wattal C. (2010) Indian J Med Microbiol, 28, 107-10.

[26] Shrivastava A., Dash P.K., Tripathi N.K., Sahni A.K., Gopalan N., Lakshmana Rao P.V. (2011) Indian J Med Microbiol, 29,51 5.

[27] Makroo R.N., Raina V., Kumar P., Kanth R.K. (2007) Asian J Transfus Sci. 1(1),4-7.

[28] Gupta E., Dar L., Kapoor G., Broor S. (2006) Virology Journal, 3,92. 